\begin{tabular}{|c|l|}
\hline Title & Platelet aggregation in citrated whole blood of the first trimester of pregnancy \\
\hline Author(s) & $\begin{array}{l}\text { Umazume, Takeshi; Y amada, Takahiro; Morikawa, Mamoru; Ishikawa, Satoshi; Furuta, Itsuko; Koyama, Takahiro; } \\
\text { Matsuno, Kazuhiko; Minakami, Hisanori }\end{array}$ \\
\hline Citation & $\begin{array}{l}\text { Clinica chimica acta, 448, 60-64 } \\
\text { https://doi.org/40.1016/.cca.2015.06.011 }\end{array}$ \\
\hline Issue Date & 2015-08-25 \\
\hline Doc URL & http://hdl.handle.net/2115/62705 \\
\hline Rights & $\begin{array}{l}\text { ○ 2015. This manuscript version is made avail lable under the CC-BY-NC-ND 4.0 license } \\
\text { http://creativecommons.org/icenses/by-nc-nd/4.0/ }\end{array}$ \\
\hline Rights(URL) & http://creativecommons.org/icenses/by-nc-nd/4.0/ \\
\hline Type & article (author version) \\
\hline File Information & manuscript.pdf \\
\hline
\end{tabular}

Instructions for use 


\section{Platelet aggregation in citrated whole blood of the first trimester of pregnancy}

Takeshi Umazume ${ }^{\mathrm{a}}$, Takahiro Yamada ${ }^{\mathrm{a}}$ * Mamoru Morikawa ${ }^{\mathrm{a}}$, Satoshi Ishikawa ${ }^{\mathrm{a}}$, Itsuko Furuta $^{\mathrm{a}}$, Takahiro Koyama ${ }^{\mathrm{a}}$, Kazuhiko Matsuno ${ }^{\mathrm{b}}$, Hisanori Minakami ${ }^{\mathrm{a}}$

a Department of Obstetrics, Hokkaido University Graduate School of Medicine, Sapporo, Japan

b Division of Laboratory and Transfusion Medicine, Hokkaido University Hospital, Sapporo, Japan

*Corresponding author:

Takahiro Yamada, MD, $\mathrm{PhD}$,

Department of Obstetrics, Hokkaido University Graduate School of Medicine, Kita-ku N14 W6, Sapporo 060-8638, Japan

TEL +81-11-706-6051 FAX +81-11-706-7981

E-mail address: taka0197@med.hokudai.ac.jp

Short title: Platelet aggregability in pregnancy 


\begin{abstract}
It was recently suggested that platelet reactivity is reduced in early pregnancy. This study was performed to determine whether the citrated whole blood from 33 pregnant women in first trimester showed spontaneous platelet aggregation and whether it differed in extent from that of 11 non-pregnant women. Platelet count and number of platelet aggregates (PA) were serially determined in the same citrated whole blood specimens at $15,30,45,60$, 75, and 90 minutes after blood sampling using a hematology analyzer. The number of PA increased significantly at 30 minutes and thereafter in both groups, but was consistently lower for pregnant than non-pregnant women over the 90 -minute observation period. The platelet count decreased significantly in a time-dependent manner in both groups, but was significantly lower at 30 and 90 minutes for non-pregnant than pregnant women. The number of PA showed a significant positive correlation with net decrease in platelet count for both pregnant and non-pregnant women. PA counts were also significantly positively correlated with mean platelet volume. In conclusion, platelet reactivity monitored by the increase in number of PA and the fall in platelet count was reduced in early pregnancy compared with non-pregnant healthy controls. (194 words)
\end{abstract}

Key words: mean platelet volume, platelet reactivity, pregnancy, spontaneous platelet aggregation, venous thromboembolism 


\section{Highlights}

- Extent of spontaneous platelet aggregation (SPA) was examined.

- Pregnant women in first trimester were compared with healthy controls.

- The SPA was less likely to occur in pregnant women.

- Fall in platelet count was greater in healthy controls.

- Thus, platelet reactivity was reduced in early pregnancy. 


\section{Introduction}

Platelets play a critical role in hemostasis and thrombosis. Therefore, detection of enhanced platelet reactivity may be clinically useful for both primary and secondary prevention of vascular events and for assessing the effectiveness of antiplatelet drugs. However, traditional platelet function tests have limited clinical application as they require blood sample processing, are time consuming, labor intensive, and require skilled laboratory staff to perform and interpret the assay.

A single platelet has a diameter of $2-4 \mu \mathrm{m}$, while activated platelets aggregate and form platelet clumps with a larger diameter. When citrated whole blood is stirred or mixed in some other way, spontaneous platelet aggregation occurs as a result of platelet activation [1-4]. A hematology analyzer (CELL-DYN Sapphire Hematology System ${ }^{\circledR}$; Abbott Diagnostics, Abbott Park, IL) [5] with a newly developed software package can specifically count the number of platelet aggregates (PA) in citrated whole blood. Spontaneous platelet aggregation is detected more frequently using this analyzer in chronic cerebral infarction patients not treated with antiplatelet drugs than in normal controls and chronic cerebral infarction patients treated with antiplatelet drugs [6]. Therefore, the number of PA detected by this analyzer is suggested to reflect the degree of platelet reactivity.

Pregnancy is associated with a hypercoagulable state with increased risk of venous thromboembolism [7,8]. Women with antiphospholipid syndrome and essential thrombocythemia are likely to experience adverse outcomes such as abortion and fetal demise $[9,10]$. These adverse pregnancy outcomes may be associated with enhanced hypercoagulable state [9]. Physiological hyperfibrinogenemia in pregnancy concomitant with possible increased platelet reactivity may partly explain the hypercoagulable state in 
pregnancy. Early studies regarding platelet parameters in pregnancy suggested enhanced platelet destruction accompanied by increased platelet production [11-14]. However, previous studies examining platelet activation during normal pregnancy yielded conflicting results - some reported increased activation $[13,15,16]$, while others reported no change $[17,18]$. In addition, a recent report suggested that platelet reactivity to collagen was

significantly reduced during the first trimester of pregnancy compared with that in non-pregnant controls [19].

The present study was performed to determine whether the citrated whole blood of pregnant women in first trimester exhibits spontaneous platelet aggregation and whether its extent differs from that in non-pregnant controls.

\section{Material and Methods}

This study was conducted after receiving approval from the Institutional Review Board of Hokkaido University Hospital and written informed consent was obtained from all participants.

\subsection{Participants}

A total of 44 healthy women consisting of 33 pregnant women in first trimester and 11 non-pregnant healthy women participated in this study and provided 44 blood specimens. None of 44 participants were positive for anti HIV-1/2 antibody and all 44 women were normotensive and had unremarkable medical histories. All 33 women in first trimester did not develop hypertensive disorders of pregnancy (HDP) and experienced uneventful pregnancies. Thus, all participants were considered healthy and not to have pathological hypercoagulability. The mean \pm SD gestational week at blood sampling was $11.0 \pm 1.0$ 
(range, $8-13$, Table 1). Age of participants did not differ significantly between the two groups.

\subsection{Blood sample collection}

Blood sampling was not performed at any particular time of the day, and without any particular time in relation to meal intake. A total of $9-10 \mathrm{~mL}$ of venous blood was drawn from the antecubital vein using a tourniquet and a 23-gauge needle connected to three successive vacuum tubes in the following order: $5-6 \mathrm{~mL}$ of blood in the first tube not used in this study, $2 \mathrm{~mL}$ of blood in the second tube containing $4.5 \mathrm{mg}$ of EDTA, and 1.8 $\mathrm{mL}$ of blood in the third tube containing $0.2 \mathrm{~mL}$ of $3.2 \%$ sodium citrate solution. Two tubes containing whole blood anticoagulated with EDTA and citrate, designated as EDTA blood and citrated blood, respectively, were used in this study.

\subsection{Measurement of platelet count and number of $P A$}

Two tubes containing EDTA blood and citrated blood were applied to the CELL-DYN Sapphire Hematology System ${ }^{\circledR}$ (Abbott Diagnostics、Abbott Park, IL) 15 minutes after blood sampling at room temperature. These tubes were agitated in this system that took approximately 3 minutes to measure platelet count, number of PA, size of immature platelet fraction (IPF), mean platelet volume (MPV), and hemoglobin concentration simultaneously. This procedure was repeated six times for each citrated blood sample to determine changes over time in number of PA and platelet count at $15,30,45,60,75$, and 90 minutes after blood sampling. For the platelet count in citrated blood, the corrected platelet count for the dilution with $0.2 \mathrm{~mL}$ of sodium citrate solution was used. Platelet count determined in the EDTA blood at 15 minutes was used as the baseline platelet count of each blood sample. The von Willebrand factor (vWF) antigen level was measured using STA ${ }^{\circledR}$ Liatest ${ }^{\circledR}$ VWF:Ag (Daignostica Stago S.A.S., Gennevilliers, France) and expressed as 
a percentage of normal activity.

\subsection{Statistics}

Data are presented as means \pm SD. Statistical analyses were performed using the JMP ${ }^{\circledR}$ Pro11 statistical software package (SAS, Cary, NC). Differences in the means were tested using the Wilcoxon rank sum test between each group, and changes in variables within a group were compared using the Wilcoxon signed-rank test. Pearson product-moment correlation coefficient was used to measure linear correlation between two variables. In all analyses, $P<0.05$ was taken to indicate statistical significance.

\section{Results}

Baseline platelet count and MPV did not differ between the two groups, while IPF and hemoglobin concentration were significantly lower and vWF antigen level was significantly higher in pregnant women than in non-pregnant women (Table 1).

Spontaneous platelet aggregation was detected in all pregnant and non-pregnant women (Fig. 1). The number of PA increased significantly at 30 minutes or later after blood sampling in both groups and was significantly greater at 30 minutes or later (except for 60 minutes $)$ in non-pregnant women than in pregnant women $(1018 \pm 1566$ vs. $196 \pm 343 / \mu \mathrm{L}$, respectively) (Fig. 2, left). Accordingly, platelet count decreased progressively in a time-dependent manner from a baseline level of $282 \pm 70$ vs. $297 \pm 60 \times 10^{3} / \mu \mathrm{L}$ to $162 \pm$ 52 vs. $116 \pm 48 \times 10^{3} / \mu \mathrm{L}$ at 90 minutes after blood sampling in pregnant and non-pregnant women, respectively (Fig. 2, right). The platelet count was significantly lower for non-pregnant women than for pregnant women at 30 minutes $(160 \pm 63$ vs. $207 \pm 58 \times$ $\left.10^{3} / \mu \mathrm{L}\right)$ and 90 minutes $\left(116 \pm 48 \mathrm{vs} .162 \pm 52 \times 10^{3} / \mu \mathrm{L}\right)$ after blood sampling. The log-transformed PA count was significantly positively correlated with net decrease in platelet count for both pregnant and non-pregnant women (Fig. 3). 
Regression analyses were performed to determine which variables determined PA counts at 15, 30, 45, 60, 75, and 90 minutes after blood sampling. Among the four variables examined (MPV, IPF, vWF, and hemoglobin concentration), only MPV was significantly positively correlated with PA counts determined at 15 minutes (Fig. 4, left), 60 minutes, and 75 minutes (Fig. 4, middle) after blood sampling, and only vWF was significantly negatively correlated with PA counts determined at 75 minutes (Fig. 4, right). The $\mathrm{r}$ and $P$ values ranged from -0.01 to -0.20 and from 0.21 to 0.96 for IPF, from -0.07 to -0.12 and from 0.45 to 0.68 for vWF vs. PA counts other than those at 75 minutes, and from - 0.01 to - 0.19 and from 0.22 to 0.97 for hemoglobin concentration, respectively.

As shown in Fig. 1, the size of a single PA varied over a wide range. Platelet counts decreased disproportionately more for the increase in number of PA in pregnant women compared with non-pregnant women (Figs. 2 and 3). Changes in mean number of single platelets forming a single PA (MNSP, number of singe platelets per single PA) were examined (Fig. 5). Surprisingly, the MNSP was markedly greater in pregnant women than in non-pregnant women; e.g., $112 \pm 134$ vs. $23 \pm 31 \times 10^{2}$ (per PA) at 15 minutes $(P=$ 0.02). In addition, the MNSP gradually decreased over the 90 -minute observation period in both groups.

\section{Discussion}

This study demonstrated that the number of PA detected by the CELL-DYN Sapphire Hematology System ${ }^{\circledR}$ was significantly lower in women in the early stage of pregnancy than in non-pregnant healthy control women, and the PA count was significantly positively correlated with both net decrease in platelet count and MPV. These results suggested that 
"PA count by our system" reflected "likelihood of spontaneous platelet aggregation," and that the platelet reactivity monitored with the increase in PA count as well as the fall in platelet count was reduced in early pregnancy compared with non-pregnant women.

A gradual decline of platelet count in the whole blood anticoagulated with citrate in this study was consistent with the results of early studies [3, 4. The platelet count gradually decreases in time-dependent manner up to two hours after blood sampling in stirred whole blood anticoagulated with citrate, but not with EDTA [3]. Fewer single platelets and aggregates containing many hundreds of cells are seen microscopically on the blood film of such citrated blood [3], and removal of adenosine diphosphate (ADP) from the blood inhibits aggregate formation $[3,4]$. The ADP that is responsible for platelet aggregates and the decrease in platelet count originates from red blood cells [4]; the extent of spontaneous platelet aggregation was quantified by the fall in number of single platelets [4]. The fall in platelet count was $56 \%$ in the citrated whole blood, but only $3 \%$ in platelet-rich plasma prepared from the same blood samples [4].

Larger platelets are more reactive, producing higher levels of ADP, $\beta$-thromboglobulin, and thromboxane $B_{2}[20-22]$, and aggregate more in response to a fixed dose of ADP and collagen [20,21]. Based on these reports [20-22], the significant positive correlation between MPV and number of PA observed in this study implied that PA count in our system may have reflected platelet reactivity. Therefore, the consistently lower PA count in addition to lower degree of fall in platelet count in the blood of pregnant women than in non-pregnant women in this study strongly suggested that platelet reactivity is reduced in early pregnancy, consistent with results of the previous study by Burke et al. [19].

Pregnancy is associated with increased platelet production and enhanced platelet 
destruction based on the following observations: platelet distribution width increases with advancing gestation $[11,14]$ and MPV versus platelet count shows a significant inverse relationship in pregnancy [14]; some pregnant women exhibit a gradual decline in platelet count in late pregnancy, especially in pregnancy complicated with HDP [23,24]; platelet lifespan is shortened in pregnancy, especially in pregnancy complicated with HDP [25]; and bone marrow megakaryocyte concentration and size are increased in late pregnancy in rats [26]. These reports led to the misunderstanding that platelet reactivity in early pregnancy is similar to that in non-pregnant women, but increases gradually with advancing gestation, especially in women complicated with HDP. Therefore, the results of the study of Burke et al. [19] indicating reduced platelet reactivity in early pregnancy was surprising. Burke et al. [19] reported that collagen-induced platelet aggregation was lowest during the first trimester, increased in the second and third trimesters and became comparable to that in non-pregnant women.

The size of PA defined as MNSP was significantly greater in pregnant women than in non-pregnant women in this study, in contrast to our expectations. We first expected greater MNSP in more enhanced platelet activity. However, platelet reactivity assessed by the increase in number of PA and the fall in platelet count suggested the decreased platelet reactivity in pregnant women compared with non-pregnant women. The present data suggested that larger numbers of single platelets were likely to aggregate together, forming large PA in the presence of decreased platelet reactivity in pregnant women. It remains unclear which factors determine the size of PA except for time after blood sampling. The MNSP became smaller over 90 minutes after blood sampling.

In conclusion, 33 pregnant women in first trimester were compared with 11 healthy non-pregnant women with respect to changes in number of PA and platelet count in 
citrated whole blood over 90 minutes after blood sampling using a hematology analyzer. The results suggested that the platelet reactivity monitored by the number of PA and the fall in platelet count was reduced in early pregnancy compared with non-pregnant healthy controls. Although mechanisms involved in this phenomenon remained to be studied, the reduced platelet reactivity in the first trimester was speculated to play an important role in the avoidance of fetal loss.

\section{Acknowledgement}

This study was supported by a Grant-in-Aid for Scientific Research from the Ministry of Education, Science, Sports, and Culture of Japan (No. 26462468). A hematology analyzer (CELL-DYN Sapphire Hematology System ${ }^{\circledR}$ ) and software package specific for data analyses of this analyzer were kindly loaned to our laboratory for this study. All of authors thank to Dr. Nobuo Masauzi and Dr. Masahiro Ieko for their helpful suggestions to this study.

\section{Disclosure}

The authors have no conflicts of interest. 


\section{References}

[1] Armstrong R, May JA, Lösche W, Heptinstall S. Factors that contribute to spontaneous platelet aggregation and streptokinase-induced aggregation in whole blood. Thromb Haemost 1995; 73: 297-303.

[2] Konstantopoulos K, Grotta JC, Sills C, Wu KK, Hellums JD. Shear-induced platelet aggregation in normal subjects and stroke patients. Thromb Haemost 1995; 74: 1329-32. [3] Fox SC, Burgess-Wilson M, Heptinstall S, Mitchell JR. Platelet aggregation in whole blood determined using the Ultra-Flo 100 Platelet Counter. Thromb Haemost 1982; 48: $327-9$.

[4] Saniabadi AR, Lowe GD, Barbenel JC, Forbes CD. A comparison of spontaneous platelet aggregation in whole blood with platelet rich plasma: additional evidence for the role of ADP. Thromb Haemost 1984; 51: 115-8.

[5] Bowen KL, Procopio N, Wystepek E, Glazier J, Mattson JC. Platelet clumps, nucleated red cells, and leukocyte counts: A comparison between the Abbott CELL-DYN® 4000 and Coulter® STKS. Lab Hematol. 1998;4:7-16.

[6] Shimizu M, Yamamoto M, Miyachi H, Shinohara Y, Ando Y. Simple, rapid, and automated method for detection of hyperaggregability of platelets using a hematology analyzer. Am J Hematol 2003; 72: 282-3.

[7] Cerneca F, Ricci G, Simone R, Malisano M, Alberico S, Guaschino S. Coagulation and fibrinolysis changes in normal pregnancy. Increased levels of procoagulants and reduced levels of inhibitors during pregnancy induce a hypercoagulable state, combined with a reactive fibrinolysis. Eur J Obstet Gynecol Reprod Biol 1997;73:31-6.

[8] Ros Salonen H, Lichtenstein P, Bellocco R, Petersson G, Cnattingius S. Increased risks of circulatory diseases in late pregnancy and puerperium. Epidemiology 2001;12:456-60. 
[9] Levy RA, Dos Santos FC, de Jesús GR, de Jesús NR. Antiphospholipid antibodies and antiphospholipid syndrome during pregnancy: Diagnostic concepts. Front Immunol 2015 May 7; 6: 205. doi: 10.3389/fimmu.2015.00205.

[10] Umazume T, Yamada T, Akaishi R, Araki N, Nishida R, Morikawa M, et al. Essential thrombocythemia as a risk factor for stillbirth. Thromb Res 2014; 133: 158-61.

[11] Fay RA, Hughes AO, Farron NT. Platelet in pregnancy: hyperdestruction in pregnancy. Obstet Gynecol 1983; 61: 238-240.

[12] Douglas JT, Shah M, Lowe GDO, Belch JJF, Forbes CD, Prentice CRM. Plasma fibrinopeptide A and beta-thromboglobulin in preeclampsia and pregnancy hypertension. Thromb Haemost 1982; 47: 54 - 55.

[13] Holmes VA, Wallace JM, Gilmore WS, McFaul P, Alexander HD. Soluble P-selectin levels during normal pregnancy: a longitudinal study. BJOG 2002; 109: 997-1002.

[14] Tygart SG, McRoyan DK, Spinnato JA, McRoyan CJ, Kitay DZ. Longitudinal study of platelet indices during normal pregnancy. Am J Obstet Gynecol 1986;154: 883-7.

[15] Gerbasi FR, Bottoms S, Farag A, Mammen E. Increased intravascular coagulation associated with pregnancy. Obstet Gynecol 1990; 75: 385 - 389 .

[16] Janes SL, Goodall AH. Flow cytometric detection of circulating activated platelets and platelet hyper-responsiveness in pre-eclampsia and pregnancy. Clin Sci 1994; 86: 731 $-739$.

[17] Romero R, Snyder E, Scott D, Oyarzun E, Hobbins JC, Duffy TP. Beta-thromboglobulin during normal pregnancy, labor, and puerperium. Am J Perinatol 1988;5:109-112.

[18] Gatti L, Tenconi PM, Guarneri D, Bertulessi C, Ossola MW, Bosco P, et al. Hemostatic parameters and platelet activation by flow-cytometry in normal pregnancy: a longitudinal study. Int J Clin Lab Res 1994;24:217 - 219. 
[19] Burke N, Flood K, Murray A, Cotter B, Dempsey M, Fay L, et al. Platelet reactivity changes significantly throughout all trimesters of pregnancy compared with the nonpregnant state: a prospective study. BJOG 2013; 120: 1599-1604.

[20] Thompson CB, Eaton KA, Princiotta SM, Rushin CA, Valeri CR. Size dependent platelet subpopulations: relationship of platelet volume to ultrastructure, enzymatic activity, and function. Br J Haematol1982; 50: 509-19.

[21] Thompson CB, Jakubowski JA, Quinn PG, Deykin D, Valeri CR. Platelet size as a determinant of platelet function.J Lab Clin Med 1983;101: 205-13.

[22] Jakubowski JA, Thompson CB, Vaillancourt R, Valeri CR, Deykin D. Arachidonic acid metabolism by platelets of differing size. Br J Haemaol 1983; 53: 503-11.

[23] Minakami H, Kuwata T, Sato I. Gestational thrombocytopenia: is it new? Am J Obstet Gynecol 1996; 175: 1676-7.

[24] Tsunoda T, Ohkuchi A, Izumi A, Watanabe T, Matsubara S, Sato I, et al. Antithrombin III activity and platelet count are more likely to decrease in twin pregnancies than in singleton pregnancies. Acta Obstet Gynecol Scand 2002; 81: 840-5.

[25] Rakocozi I, Tallian F, Bagdany S, Gati I. Platelet life-span in normal pregnancy and pre-eclampsia as determined by a non-radioisotope technique. Thromb Res 1979;15: $553-556$.

[26] Jackson CW, Steward SA, Ashmun RA, McDonald TP. Megakaryocytopoiesis and platelet production are stimulated during late pregnancy and early postpartum in the rat. Blood 1992: 79: 1672-1678. 


\section{Figure Captions}

Fig. 1. Representative scatter plots by the CELL-DYN in two blood samples

$\mathrm{A}$ and $\mathrm{B}$, Blood 30 minutes after sampling from a pregnant woman and a non-pregnant woman, respectively. Each platelet aggregate (PA) is shown as a tiny white dot. Lymphocytes and neutrophils are shown in blue and yellow, respectively.

Fig. 2. Changes in number of $P A$ and platelet count according to time after blood sampling

Black and red lines with vertical bars indicate mean number of PA with SD for non-pregnant women $(n=11)$ and pregnant women in first trimester $(n=33)$, respectively. Baseline platelet count (at 0 minutes) was that determined in the EDTA blood 15 minutes after sampling.

Fig. 3. Correlation between log-transformed PA count and net decrease in platelet count

A, 198 measurements in 33 pregnant women; B, 66 measurements in 11 non-pregnant women.

Fig. 4. Correlation between log-transformed PA counts and mean platelet volume (MPV) and von Willebrand Factor (vWF) levels

There were statistically significant positive correlations between MPV and log-transformed PA count at 15 minutes (left), 60 minutes (not shown in this figure), and 75 minutes (middle) after blood sampling. $\mathrm{r}=0.37, P=0.014$ for PA count at 60 minutes after blood sampling. There was a statistically significant negative correlation between vWF and log-transformed PA count at 75 minutes (right) after blood sampling. 
Fig. 5. Changes in estimated number of single platelets forming a single SPA MNSP, mean number of single platelets forming a single PA. The MNSP at N minutes was estimated using the following equation: net decrease in platelet count during $\mathrm{N}$ minutes after blood sampling divided by number of PA at $\mathrm{N}$ minutes. 
Fig. 1.

A

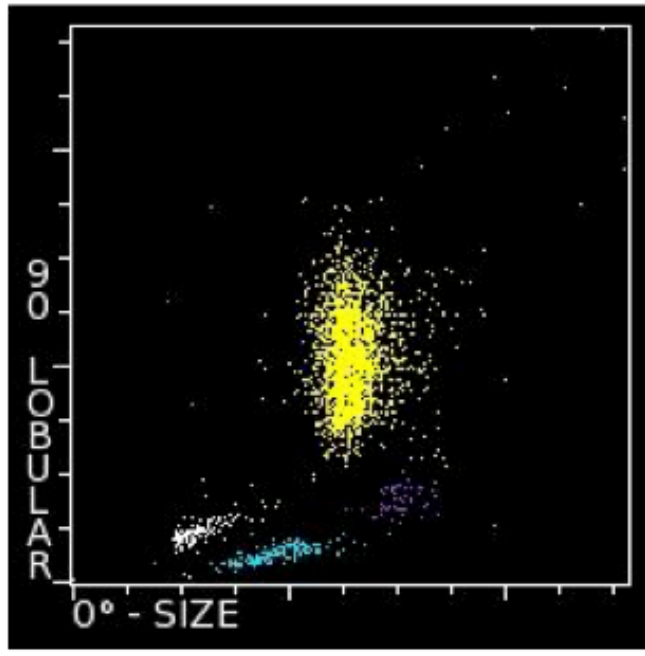

B

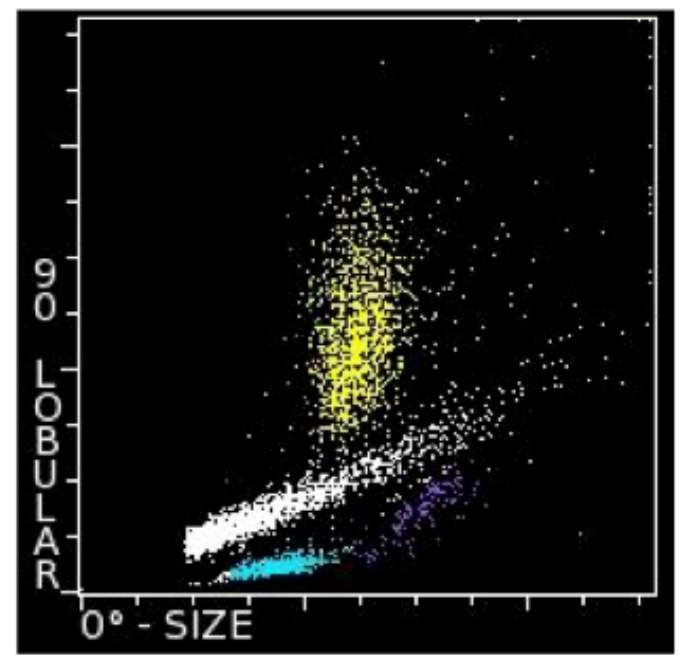

Fig. 2.
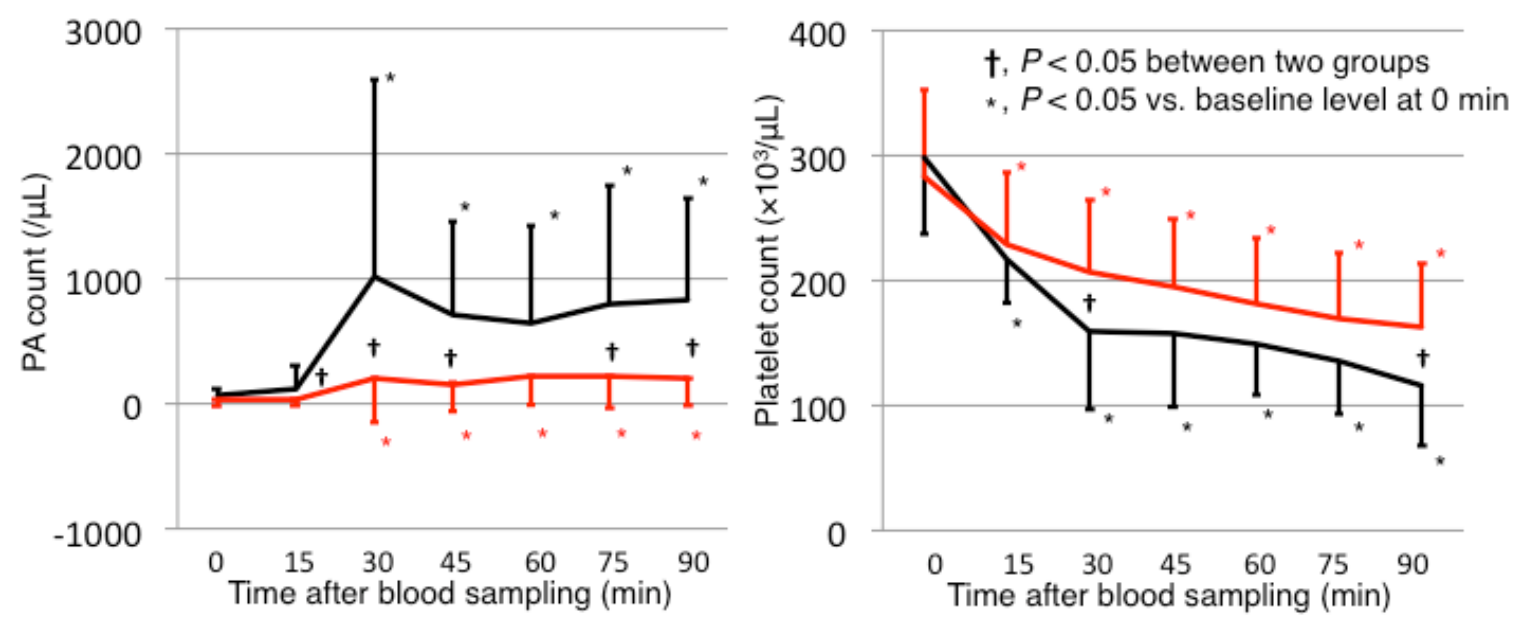
Fig. 3.

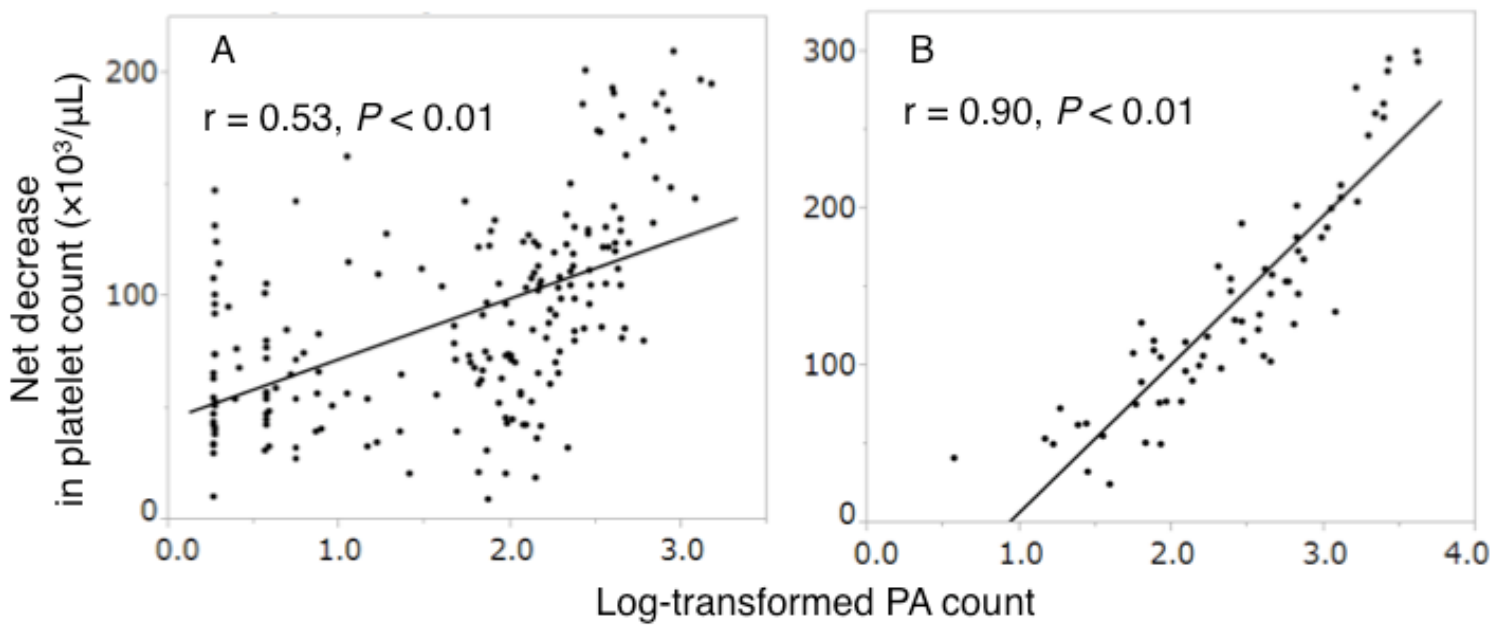

Fig. 4.
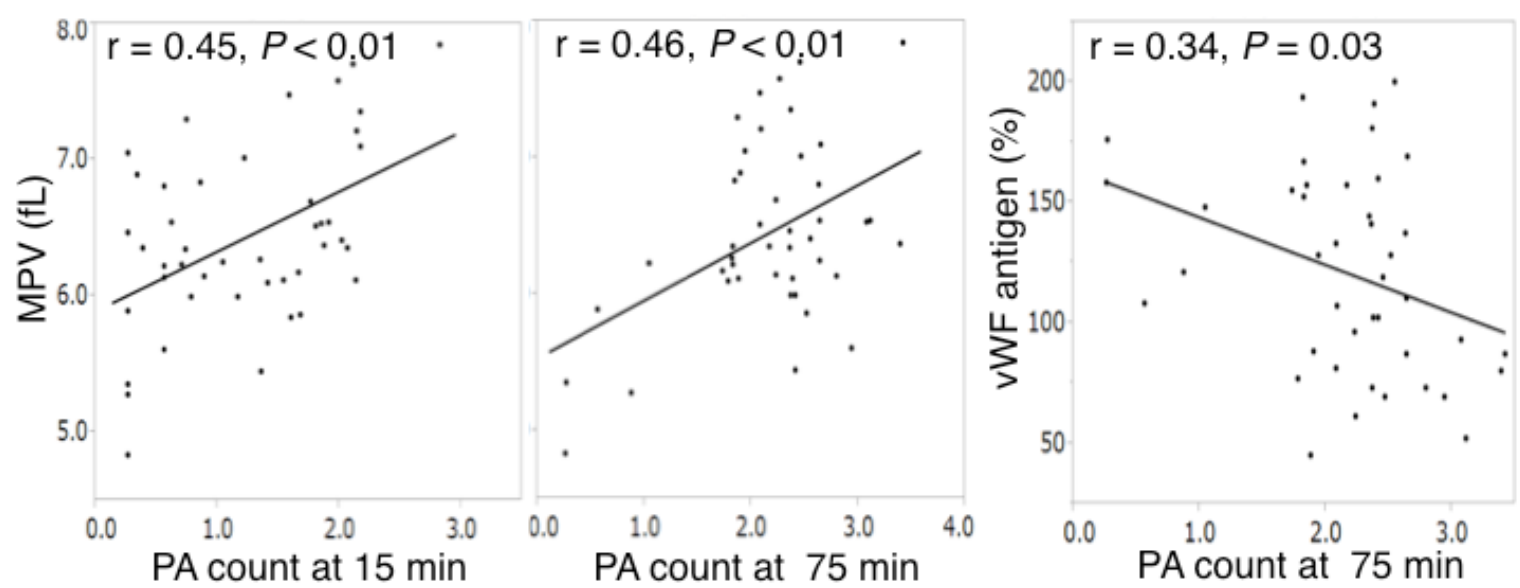
Fig. 5.

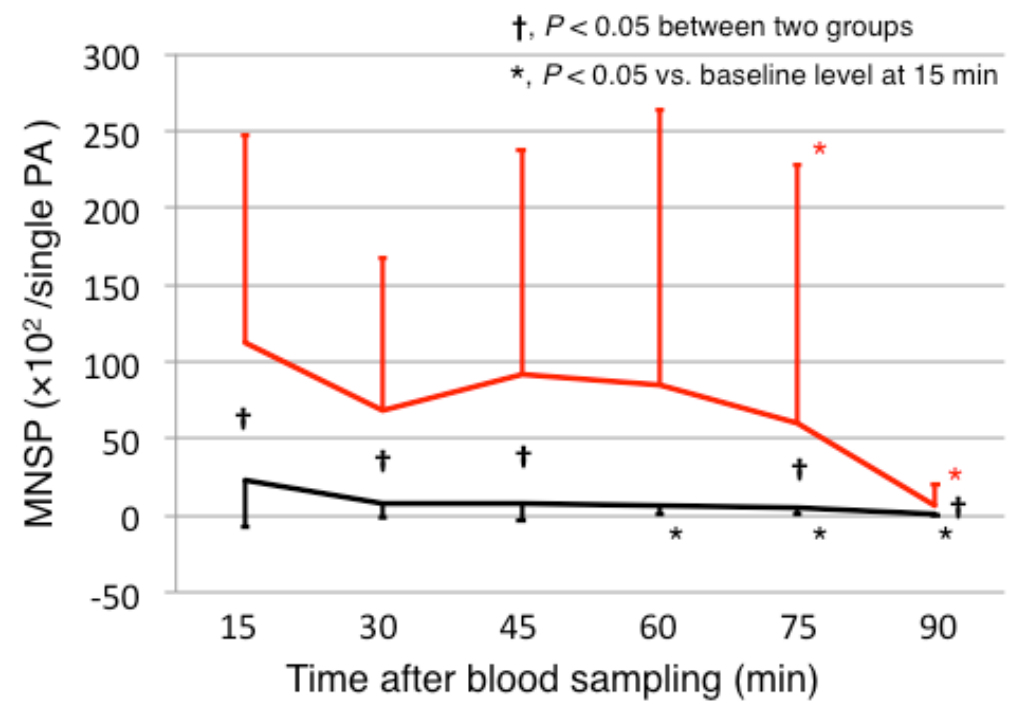


Table I. Characteristics of 33 pregnant women and 11 non-pregnant women

\begin{tabular}{lcll}
\hline & Pregnant $(n=33)$ & Non-pregnant $(n=11)$ & $P$-value \\
\hline Age $($ year $)$ & $32.2 \pm 4.6$ & $31.3 \pm 4.8$ & 0.49 \\
BMI $\left(\mathrm{kg} / \mathrm{m}^{2}\right)$ & $22.4 \pm 5.5$ & $20.8 \pm 2.8$ & 0.49 \\
Gestational week & $11.0 \pm 1.0$ & NA & \\
Platelet count* $\left(\times 10^{3} / \mu \mathrm{L}\right)$ & $282 \pm 70$ & $297 \pm 60$ & 0.38 \\
MPV (fL) & $6.30 \pm 0.63$ & $6.67 \pm 0.61$ & 0.15 \\
IPF $(\%)$ & $3.5 \pm 2.1$ & $4.6 \pm 2.0$ & 0.04 \\
vWF antigen $(\%)$ & $130 \pm 35$ & $92 \pm 47$ & $<0.01$ \\
Hemoglobin $(\mathrm{g} / \mathrm{dL})$ & $12.7 \pm 0.9$ & $13.4 \pm 1.8$ & $<0.01$ \\
Data are presented as means \pm SD. BMI, body mass index; IPF, immature platelet fraction; MPV, mean \\
platelet volume; NA, not applicable; vWF, von Willebrand Factor; *, determined in EDTA blood samples \\
15 min after blood sampling.
\end{tabular}

